\title{
ASYMPTOTIC PROPERTIES OF SUBHARMONIC FUNCTIONS OF ORDER LESS THAN ONE
}

\section{JUN-ITI ITÔ}

1. Introduction. Let $u(z)$ be a subharmonic function in the finite plane, $M(r)$ the maximum of $u(z)$ for $|z|=r>0$, and $m(r)$

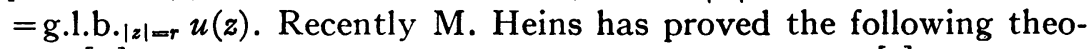
rem [4] as a precision of the classical Wiman theorem [8].

TheOREM 1. Let $u(z)$ be not identically constant and for all nonnegative $r, m(r) \leqq A<\infty$. Suppose that $\lim \inf _{r \rightarrow \infty} r^{-1 / 2} M(r)<\infty$. Then

$$
\lim _{r \rightarrow \infty} r^{-1 / 2} M(r)
$$

exists (a positive finite limit).

The purpose of this paper is to study how much the condition that $m(r) \leqq A<\infty$ for all $r>0$ in the above theorem can be weakened without destroying the existence of the limit (1.1). The principal theorem is as follows:

TheOREm 2. Let $u(z)$ be a subharmonic function. Suppose that ${ }^{1}$

$$
\int_{r_{1}}^{r_{2}} r^{-3 / 2} m(r) d r<\epsilon
$$

for all $r_{2}>r_{1}>N$ if $N$ is selected sufficiently large for an arbitrary positive $\epsilon$ and

$$
\limsup _{r \rightarrow \infty} r^{-1 / 2} M(r)<\infty .
$$

Then (1.1) exists (a non-negative finite limit).

We remark that each of the conditions

$$
\int_{1}^{\infty} r^{-3 / 2} m(r) d r \text { exists }
$$

and

$$
\int_{1}^{\infty}\left(m^{+}(r) /(1+r) r^{1 / 2}\right) d r<\infty
$$

implies the condition (a). Therefore the hypothesis (a) of Theorem 2

Received by the editors July 18, 1957 and, in revised form, December 3, 1957.

1 Throughout this paper, integral means $L$-integral. 
can be replaced by the condition (c) or (d).

If $\lim \sup _{r \rightarrow \infty} r^{-\rho} M(r)<\infty$ for some positive $\rho<1, u(z)$ is said to be of order less than one. Let $u(z)$ be of order less than one and harmonic at $z=0$. Then Heins [4] has proved the following representation theorem. "There exists a generalized positive mass distribution $\mu(e)$ defined for Borel set $e$ in the finite plane such that

$$
u(z)=u(0)+\int \log \left|1-\frac{z}{\zeta}\right| d \mu\left(e_{\zeta}\right)
$$

for all finite $z . "$

Let $\mu^{*}(t)=\mu[|z|<t]$ for $t>0$. Then Theorem 2 is also generalized as follows:

THEOREM 3. Let for some positive $0<\rho<1$,

$$
\begin{aligned}
& \int_{r_{1}}^{r_{2}} r^{-1-\rho}\left\{m(r) \sin \rho \pi-\pi \mu^{*}(r) \cos \rho \pi\right\} d r<\epsilon \\
& \text { for all } r_{2}>r_{1}>N
\end{aligned}
$$

if $N$ is selected sufficiently large for an arbitrary positive $\epsilon$ and

$$
\alpha=\limsup _{r \rightarrow \infty} r^{-\rho} M(r)<\infty .
$$

\section{Then}

$$
\lim _{r \rightarrow \infty} r^{-\rho} M(r)
$$

exists (a non-negative finite limit).

Clearly Theorem 3 implies Theorem 2. Moreover

THEOREM 4. Let for some positive $0<\rho<1$,

$$
\int_{1}^{\infty} t^{-1-\rho}\left\{m(t)-t^{\rho} \cos \rho \pi\right\}+d t<\infty .
$$

If either (1) $1>\rho>1 / 2, \lim \sup _{r \rightarrow \infty} r^{-1} M(r) \leqq 0$ and

$$
\int_{1}^{\infty} t^{-1-\rho}\left\{\pi \mu^{*}(t)-t^{\rho} \sin \rho \pi\right\}+d t<\infty
$$

or (2) $1 / 2>\rho>0, \lim \sup _{r \rightarrow \infty} r^{-1 / 2} M(r) \leqq 0$ and

$$
\int_{1}^{\infty} t^{-1-\rho}\left\{-\pi \mu^{*}(t)+t^{\rho} \sin \rho \pi\right\}+d t<\infty,
$$




$$
\lim _{r \rightarrow \infty} r^{-\rho} M(r)=1 .
$$

The Heins' theorem [4, Theorem E], which is related to Variron's theorem, is generalized as follows:

TheOREм 5. If a function $u(z)$ satisfying the hypotheses of Theorem 3 satisfies $\alpha>0$,

$$
\mu^{*}(r) \sim \frac{\alpha}{\pi} r^{\rho} \sin \rho \pi
$$

THEOREM 6. For a function satisfying the hypotheses of Theorem 4,

$$
\mu^{*}(r) \sim \frac{1}{\pi} r^{\rho} \sin \rho \pi .
$$

2. Proof of Theorem 3. If $u(z)$ is a subharmonic function and satisfies the condition ( $f$ ),

$$
\mu^{*}(r)=O\left(r^{\rho}\right) .
$$

Without loss of generality we may assume that $u(z)$ is actually harmonic at $z=0$ and $u(0)=0$. Let $w(z)$ and $S(z)$ be defined respectively, with $\arg w(z)=0$ on the positive real axis, by

$$
\begin{aligned}
w(z)=u_{1}(z)+i v_{1}(z) & =\int_{0}^{\infty} \log \left(1+\frac{z}{t}\right) d \mu^{*}(t), \\
S(z) & =U(z)+i V(z)=z^{1 / 2-\rho} w(z)
\end{aligned}
$$

where $u_{1}(z), v_{1}(z), U(z)$ and $V(z)$ denote real functions of $z$ respectively. Then $w(z)$ and $S(z)$ are regular in $|\arg z|<\pi$ and

(2.4) $U(z)=|z|^{1 / 2-\rho}\left\{u_{1}(z) \cos (\rho-1 / 2) \theta+v_{1}(z) \sin (\rho-1 / 2) \theta\right\}$.

Therefore

$$
\int_{-\pi}^{+\pi}\left|U\left(r e^{i \theta}\right)\right| d \theta=O\left(r^{1 / 2}\right) .
$$

$u_{1}(z)$ is an upper semi-continuous function on the negative real axis and $v_{1}(z)$ is continuous except for at most a countable set on the negative real axis. If we define

$$
v_{1}(-r)=\pi \mu^{*}(r)
$$

at the points at which $v_{1}(z)$ is discontinuous,

$$
U(-r)=r^{1 / 2-\rho}\left\{u_{1}(-r) \sin \rho \pi-\pi \mu^{*}(r) \cos \rho \pi\right\}
$$


and $U(-r)$ is $L$-integrable in the finite interval of $r \geqq 0$. Using (1.2) and (2.2)

$$
\begin{aligned}
U(-r) & \leqq r^{1 / 2-\rho}\left\{m(r) \sin \rho \pi-\pi \mu^{*}(r) \cos \rho \pi\right\}, \\
U(r) & =r^{1 / 2-\rho} u_{1}(r) \geqq r^{1 / 2-\rho} M(r) .
\end{aligned}
$$

On the other hand, by virtue of F. and R. Nevanlinna's representation formula for a semi-circle [5], we easily obtain the following representation of $U(z)$ such that for $z=r e^{i \theta}$ in the semi-circle

$$
\begin{aligned}
u_{2}(z) & =\frac{1}{\pi} \int_{-\pi}^{+\pi} \frac{U\left(R e^{i \phi}\right) R^{1 / 2} r^{1 / 2}(R-r) \cos \frac{\theta}{2} \cos \frac{\phi}{2} d \phi}{\left(R+r-2 R^{1 / 2} r^{1 / 2} \cos \frac{\phi-\theta}{2}\right)\left(R+r+2 R^{1 / 2} r^{1 / 2} \cos \frac{\phi+\theta}{2}\right)} \\
& =\frac{1}{\pi} \int_{-\pi}^{+\pi} U\left(R e^{i \phi}\right) K_{1}\left(R e^{i \theta}, r e^{i}\right) d \phi, \\
u_{3}(z) & =\frac{1}{\pi} \int_{0}^{R} U(-t) r^{-1 / 2} r^{1 / 2} \cos \frac{\theta}{2}\left\{\begin{array}{l}
1 \\
t+r-2 t^{1 / 2} r^{1 / 2} \sin \frac{\theta}{2}
\end{array}\right.
\end{aligned}
$$

$$
=\frac{1}{\pi} \int_{0}^{R} U(-t) K_{2}(-t, z) d t
$$

$$
\left.-\frac{R}{R^{2}+t r-2 R t^{1 / 2} r^{1 / 2} \sin \frac{\theta}{2}}\right\} d t
$$

(2.11) $U(z)=u_{2}(z)+u_{3}(z)$.

Let

$$
N\left(\rho_{1}\right)=\frac{1}{2 \pi} \int_{-\pi}^{+\pi} U\left(\rho_{1} e^{i \phi}\right) \cos \frac{\phi}{2} d \phi,
$$

from $(2.9)$

$$
\begin{aligned}
\frac{1}{2 \pi \rho^{1 / 2}} \int_{-\pi}^{+\pi} & \pi_{2}\left(\rho_{1} e^{i \theta}\right) \cos \frac{\theta}{2} d \theta \\
& =\frac{1}{2 \pi^{2} \rho^{1 / 2}} \int_{-\pi}^{+\pi} \int_{-\pi}^{+\pi} U\left(R e^{i \phi}\right) K_{1}\left(R e^{i \phi}, \rho_{1} e^{i \theta}\right) \cos \frac{\theta}{2} d \theta d \phi \\
& =\frac{1}{2 \pi^{2}} \int_{-\pi}^{+\pi} U\left(R e^{i \phi}\right) \int_{-\pi}^{+\pi} \frac{\cos \theta / 2}{\rho_{1}^{1 / 2}} K_{1}\left(R e^{i \phi}, \rho_{1} e^{i \theta}\right) d \theta d \phi \\
& =\frac{1}{2 \pi R^{1 / 2}} \int_{-\pi}^{+\pi} U\left(R e^{i \phi}\right) \cos \frac{\phi}{2} d \phi=\frac{N(R)}{R^{1 / 2}} .
\end{aligned}
$$

As $U\left(r e^{i \theta}\right)$ is $L$-integrable on intervals $[-\pi \leqq \theta \leqq \pi]$ and $[0 \leqq r \leqq R$, $\theta=\pi]$, for $\epsilon>0$ 
$\lim _{\epsilon \rightarrow 0} \frac{1}{2 \pi \rho_{1}^{1 / 2}} \int_{-\pi+\epsilon}^{\pi-\epsilon} u_{3}\left(\rho_{1} e^{i \theta}\right) \cos \frac{\theta}{2} d \theta$

$$
\begin{aligned}
& =\lim _{\epsilon \rightarrow \infty} \frac{1}{2 \pi^{2} \rho_{1}^{1 / 2}} \int_{0}^{R} U(-t) \int_{-\pi+\epsilon}^{\pi-\epsilon} K_{2}\left(-t, \rho_{1} e^{i \theta}\right) \cos \frac{\theta}{2} d \theta d t \\
& =\frac{1}{2 \pi}\left\{\frac{1}{\rho_{1}} \int_{0}^{\rho_{1}} \frac{U(-t)}{t^{1 / 2}} d t+\int_{\rho_{1}}^{R} \frac{U(-t)}{t^{3 / 2}} d t-\int_{0}^{R} \frac{U(-t)}{R t^{1 / 2}} d t\right\} .
\end{aligned}
$$

Thus we have

$$
\begin{aligned}
\frac{N\left(\rho_{1}\right)}{\rho_{1}^{1 / 2}}= & \frac{1}{2 \pi}\left\{\int_{0}^{\rho_{1}} \frac{U(-t)}{\rho_{1} t^{1 / 2}} d t+\int_{\rho_{1}}^{R} \frac{U(-t)}{t^{3 / 2}} d t-\int_{0}^{R} \frac{U(-t)}{R t^{1 / 2}} d t\right\} \\
& +\frac{N(R)}{R^{1 / 2}} .
\end{aligned}
$$

If we assume (e), from (2.7)

$$
\int_{\sigma_{1}}^{\sigma_{2}} \frac{U(-t)}{t^{3 / 2}} d t<\epsilon
$$

for all $\sigma_{2}>\sigma_{1}>N$ if $N$ is selected sufficiently large for an arbitrary positive $\epsilon$. Next, an application of the second mean value theorem shows that for $\rho_{1}<R^{\prime}<R$ and $0<M<M^{\prime}<\rho_{1}$

$$
\begin{aligned}
\tau\left(\rho_{1}, R\right)= & \int_{0}^{\rho_{1}} \frac{U(-t)}{t^{1 / 2}}\left(\frac{1}{\rho_{1}}-\frac{1}{R}\right) d t+\int_{\rho_{1}}^{R} \frac{U(-t)}{t^{1 / 2}}\left(\frac{1}{t}-\frac{1}{R}\right) d t \\
= & \int_{0}^{M} \frac{U(-t)}{t^{1 / 2}}\left(\frac{1}{\rho_{1}}-\frac{1}{R}\right) d t+\frac{R-\rho_{1}}{R} \int_{M^{\prime}}^{\rho_{1}} \frac{U(-t)}{t^{3 / 2}} d t \\
& +\frac{R-\rho_{1}}{R} \int_{\rho_{1}}^{R^{\prime}} \frac{U(-t)}{t^{3 / 2}} d t .
\end{aligned}
$$

Thus from (2.15) and (2.16), we get

$$
\tau\left(\rho_{1}\right)=\limsup _{R \rightarrow \infty} \tau\left(\rho_{1}, R\right)<\infty,
$$

$$
\limsup _{\rho_{1} \rightarrow \infty} \tau\left(\rho_{1}\right) \leqq 0 .
$$

Hence, if $\lim \inf _{R \rightarrow \infty} R^{-1 / 2} N(R)=\mu$, from (2.5), $|\mu|<\infty$ and we can select a sequence $\left\{R_{n}\right\}$ of $R$ such that $R_{n} \rightarrow \infty$ as $n \rightarrow \infty$ and get $\lim _{n \rightarrow \infty} R_{n}^{-1 / 2} N\left(R_{n}\right)=\mu$. If for such a sequence $\left\{R_{n}\right\}$, we write $\nu\left(\rho_{1}\right)$ $=\lim _{n \rightarrow \infty} \tau\left(\rho_{1}, R_{n}\right)$, then $\nu\left(\rho_{1}\right) \leqq \tau\left(\rho_{1}\right)$. So that 


$$
\underset{\rho_{1} \rightarrow \infty}{\lim \sup } \nu\left(\rho_{1}\right) \leqq \lim _{\rho_{1} \rightarrow \infty} \sup \tau\left(\rho_{1}\right) .
$$

From (2.14), (2.17) and (2.18), $\lim \sup _{\rho_{1} \rightarrow \infty} \rho_{1}^{-1 / 2} N\left(\rho_{1}\right) \leqq \mu$. Hence

$$
\mu=\lim _{R \rightarrow \infty} R^{-1 / 2} N(R)
$$

exists (a finite limit). So that from (2.14) and (2.19)

$$
\lim _{R \rightarrow \infty} \int_{1}^{R} \frac{U(-t)}{t^{1 / 2}}\left(\frac{1}{t}-\frac{1}{R}\right) d t
$$

exists. Select $N$ sufficiently large for an arbitrary $\epsilon>0$, then for $r_{2}>r_{1}>N$

$$
\left|\int_{1}^{r_{2}} \frac{U(-t)}{t^{1 / 2}}\left(\frac{1}{t}-\frac{1}{r_{2}}\right) d t-\int_{1}^{r_{1}} \frac{U(-t)}{t^{1 / 2}}\left(\frac{1}{t}-\frac{1}{r_{1}}\right) d t\right|<\epsilon .
$$

Thus for $M<r_{1}^{\prime}<r_{1}$ and $r_{1}<r_{2}^{\prime}<r_{2}$

$$
\begin{aligned}
-\epsilon-\int_{1}^{M} \frac{U(-t)}{t^{1 / 2}}\left(\frac{1}{r_{1}}\right. & \left.-\frac{1}{r_{2}}\right) d t-\frac{r_{2}-r_{1}}{r_{2}} \int_{r_{1^{\prime}}}^{r_{1}} \frac{U(-t)}{t^{3 / 2}} d t \\
& \leqq \int_{r_{1}}^{r_{2}} \frac{U(-t)}{t^{3 / 2}} d t \leqq \epsilon+\int_{r_{2^{\prime}}}^{r^{2}} \frac{U(-t)}{t^{3 / 2}} d t .
\end{aligned}
$$

If we select $N$ sufficiently large for an arbitrary $\epsilon>0$, then from (2.15) and (2.21), for $r_{2}>r_{1}>N$

$$
\left|\int_{r_{1}}^{r_{2}} \frac{U(-t)}{t^{3 / 2}} d t\right|<\epsilon .
$$

Hence

$$
\lim _{R \rightarrow \infty} \int_{1}^{R} \frac{U(-t)}{t^{3 / 2}} d t
$$

exists. By (2.5), (2.9) and (2.19) we have

$$
u_{2}(z)=2 \mu r^{1 / 2} \cos \frac{\theta}{2} \text {. }
$$

And from (2.10) and (2.22) we get for $|\arg z|<\pi-\delta, \pi>\delta>0$, uniformly $\lim _{z \rightarrow \infty} u_{3}(z) / R z^{1 / 2}=0$ [1]. Thus from (2.11), for $|\arg z|$ $<\pi-\delta, \pi>\delta>0$, uniformly $\lim _{z \rightarrow \infty} U(z) / R z^{1 / 2}=2 \mu$. So that from (2.4)

$$
\lim _{r \rightarrow \infty} r^{-\rho} u_{1}(r)=2 \mu \text {. }
$$


Thus from (2.2), $\mu \geqq 0$. If we write

$$
T(r)=m(r)-u_{1}(-r),
$$

then $T(r) \geqq 0$. Therefore $\int_{1}^{R}\left(T(r) / r^{1+\rho}\right) d r$ is a monotonously increasing function of $R$. So that by (e) and (2.22), $\int_{1}^{\infty}\left(T(r) / r^{1+\rho}\right) d r$ exists (a finite limit). Thus selecting a monotone decreasing sequence $\left\{\epsilon_{k}\right\}$ such that $\epsilon_{k} \rightarrow 0$ as $k \rightarrow \infty$ and taking a suitable large $r_{k}$ for each $\epsilon_{k}$, we have

$$
\int_{r_{k}}^{\infty} \frac{T(r)}{r^{1+\rho}} d r<\frac{\epsilon_{k}}{2^{k}}
$$

Accordingly if $E\left(r_{k}\right)$ denotes an open set of $r$ such that $T(r)>r^{\rho} \epsilon_{k}$, for $r \geqq r_{k}$

$$
\int_{E\left(r_{k}\right)} d \log r<\frac{1}{2^{k}} .
$$

The logarithmic length of $U_{k>N}\left(r_{k}\right)$ is less than $2^{1-N}$. Thus

$$
\lim _{r \rightarrow \infty} T(r) / r^{\rho}=0
$$

except for an open set $E$ of $r$ of finite logarithmic length. On the other hand, from the definition of $u_{1}(z)$ in (2.2) and the equality of (1.2)

$$
\begin{aligned}
u(z)+u(-z) & \geqq u_{1}(|z|)+u_{1}(-|z|), \\
M(r) & \geqq u_{1}(r)+u_{1}(-r)-m(r) .
\end{aligned}
$$

By (2.26) and (2.27), we get

$$
\liminf _{r \rightarrow \infty} M(r) / r^{\rho} \geqq \lim _{r \rightarrow \infty} u_{1}(r) / r^{\rho}
$$

except for the open set $E$. Since $M(r)$ is a monotone increasing function of $r$ and $\lim _{r \rightarrow \infty} r^{-\rho} u_{1}(r)=2 \mu$ by (2.24), we obtain

$$
\liminf _{r \rightarrow \infty} M(r) / r^{\rho} \geqq 2 \mu .
$$

On the other hand, from (2.2), $u_{1}(r) \geqq M(r)$. Hence

$$
\lim _{r \rightarrow \infty} M(r) / r^{\rho}=2 \mu \text {. }
$$

3. Proof of Theorem 4. Let $u(Z)$ be a subharmonic function and $\lim \sup _{r \rightarrow \infty} r^{-1 / 2} M(r) \leqq 0$. Then the following result is known [2]: $w(z)$ in (2.2), with arg $w(z)=0$ on the positive real axis, can be written again in the form 


$$
w(z)=u_{1}(z)+i v_{1}(z)=\frac{1}{\pi} \int_{0}^{\infty} \frac{z^{1 / 2} u_{1}(-t)}{t^{1 / 2}(t+z)} d t .
$$

Hence for $0<\rho<1 / 2$

$$
u_{1}(r)-r^{\rho}=\frac{1}{\pi} \int_{0}^{\infty} \frac{r^{1 / 2}\left(u_{1}(-t)-t^{\rho} \cos \rho \pi\right)}{t^{1 / 2}(t+r)} d t .
$$

By the condition (g) and $m(r) \geqq u_{1}(-r)$,

$$
\begin{aligned}
\int_{0}^{\infty} \frac{\left(u_{1}(r)-r^{\rho}\right)^{+}}{r^{1+\rho}} & d r \\
& \leqq \frac{1}{\pi} \int_{0}^{\infty} \int_{0}^{\infty} \frac{r^{-1 / 2-\rho}\left(u_{1}(-t)-t^{\rho} \cos \rho \pi\right)^{+}}{t^{1 / 2}(t+r)} d t d r \\
& \leqq \frac{1}{\cos \rho \pi} \int_{0}^{\infty} \frac{\left(u_{1}(-t)-t^{\rho} \cos \rho \pi\right)^{+}}{t^{1+\rho}} d t<\infty .
\end{aligned}
$$

So that $\lim \sup _{r \rightarrow \infty} u_{1}(r) / r^{\rho} \leqq 1$ except for the open set of $r$ of finite logarithmic length. Hence for every $r$

$$
\underset{r \rightarrow \infty}{\lim \sup } u_{1}(r) / r^{\rho} \leqq 1 .
$$

On the other hand, from (2.2)

$$
-u_{1}(r)+r^{\rho}=\int_{0}^{\infty} \frac{r\left(-\pi \mu^{*}(t)+t^{\rho} \sin \rho \pi\right)}{\pi t(t+r)} d t .
$$

Using the above method, by the condition (i) we find

$$
\underset{r \rightarrow \infty}{\liminf } u_{1}(r) / r^{\rho} \geqq 1
$$

Hence from (3.4) and (3.6)

$$
\lim _{r \rightarrow \infty} u_{1}(r) / r^{\rho}=1
$$

From (3.3) and (3.5)

$$
\int_{0}^{\infty} \frac{u_{1}(r)-r^{\rho}}{r^{1+\rho}} d r
$$

exists (a finite limit). Therefore from (3.2), (3.3) and (3.8),

$$
\int_{0}^{\infty} \int_{0}^{\infty} \frac{r^{-1 / 2-\rho}}{t^{1 / 2}(t+r)}\left(-u_{1}(-t)+t^{\rho} \cos \rho \pi\right)+d t d r<\infty .
$$


By the condition (g) and (3.2),

$$
\int_{0}^{\infty} \frac{\left(-u_{1}(-t)+t^{\rho} \cos \rho \pi\right)^{+}}{t^{3 / 2}} d t<\infty .
$$

Hence by changing the sequence of double integral in (3.9) we have

$$
\int_{0}^{\infty} \frac{\left(-u_{1}(-t)+t^{\rho} \cos \rho \pi\right)^{+}}{t^{1+\rho}} d t \text { exists (a finite limit). }
$$

Thus from (3.7) and (3.10), using the same method as the proof of Theorem 2, we get (1.1) for $0<\rho<1 / 2$. For $1 / 2<\rho<1$ the proof is given analogously by the use of the following representation of $w(z)$ in (2.2):

$$
w(z)=\frac{-1}{\pi} \int_{0}^{\infty} \frac{z^{3 / 2} u_{1}(-t)}{t^{3 / 2}(t+z)} d t
$$

with $\arg w(z)=0$ on the positive real axis [2]. Thus the proof of this theorem is completed.

4. Proof of Theorems 5 and 6 . We consider $u_{1}(Z)$ of $(2.2)$ under the hypothesis of (2.24). $u_{1}(z)$ is the real part of the following function

$$
W(z)=z \int_{0}^{\infty} \frac{\mu^{*}(t)}{t(t+z)} d t
$$

which is regular in the domain $|\arg z|<\pi$. Therefore by virtue of the study of Heins [4], for $\mu>0$,

$$
\lim _{r \rightarrow \infty} \frac{\mu^{*}(r)}{r^{\rho}}=\frac{2 \mu}{\pi} \sin \rho \pi .
$$

From (2.28) and (4.2) we get the proof of Theorem 5.

With the same method, we find the proof of Theorem 6 by the use of the equality (3.7).

\section{REFERENCES}

1. R. P. Boas, Jr., Asymptotic properties of functions of exponential type, Duke Math. J. vol. 20 (1953) pp. 433-448.

2. N. A. Bowen and A. J. Macintyre, Some theorems on integral functions with negative zeros, Trans. Amer. Math. Soc. vol. 70 (1951) pp. 114-126.

3. M. Heins, On the Phragmen-Lindelöf principle, Trans. Amer. Math. Soc. vol. 60 (1946) pp. 238-244.

4. - Entire functions with bounded minimum modulus: Subharmonic function analogues, Ann. of Math. vol. 49 (1948) pp. 200-213.

5. F. and R. Nevanlinna, Über die Eigenshaften analytischer Funktionen in der 
Umgebung einer singulären Stelle oder Linie, Acta Societatis Scientiarum Fennicae, vol. 50 (1922).

6. R. Nevanlinna, Eindeutige analytische Funktionen, Berlin, 1936.

7. E. C. Titchmarsh, On integral functions with real negative zeros, Proc. London Math. Soc. (2) vol. 26 (1927) pp. 185-200.

8. - Theory of functions, 2d ed., Oxford, 1939.

Nagoya Institute of Technology

\section{CONVERGENCE OF SEQUENCES OF COMPLEX TERMS DEFINED BY ITERATION}

\section{A. G. AZPEITIA}

In a paper by J. Aczel [1], the following result is established: Let $m\left(x_{1}, x_{2}, \cdots, x_{p}\right)$ be a continuous real function of the real variables $x_{i}\left(i=1,2, \cdots, p,-\infty<a<x_{i}<b<+\infty\right)$ such that: (a) $m(x, x, \cdots, x)=x$ for any $x$ in $(a, b)$, and (b) $m\left(x_{1}, x_{2}, \cdots, x_{p}\right)$ is strictly increasing with respect to all $x_{i}$. Then the sequence $a_{n}$ $=m\left(a_{n-p}, a_{n-p-1}, \cdots, a_{n-1}\right)$ with the initial terms $a_{1}, a_{2}, \cdots, a_{p}$ arbitrarily chosen in $(a, b)$ is convergent.

As corollaries of this theorem, the author proves the theorem of Eneström and Kakeya, ${ }^{1}$ and some well known elementary results. ${ }^{2}$ In fact, the theorem of Eneström and Kakeya is proved to be equivalent to the Aczel result if the function $m$ is of the form

$$
m\left(x_{1}, x_{2}, \cdots, x_{p}\right)=\left(\sum_{i=1}^{p} c_{i} x_{i}\right) /\left(\sum_{i=1}^{p} c_{i}\right), \quad\left(c_{i}>0\right) .
$$

In the present paper, we prove the following more general theorem for the case of complex variables. The nature of the proof is necessarily new because of the impossibility of establishing any relations of inequality for the complex numbers similar to the ones used by Aczel in his proof. The concept of convexity is used to overcome this difficulty and no assumption of analyticity is required.

Received by the editors July 13, 1957 and, in revised form, October 2, 1957 and November 15, 1957.

1 The simplest statement of this theorem is: Let $z$ be any root of $\sum_{j=0}^{m} b_{j} z^{i}=0$ with $b_{m}, b_{m-1}, \cdots, b_{0}$ decreasing positive real numbers. Then, $z$ is of an absolute value less than 1. (See $[2 ; 3 ; 4]$.)

${ }^{2}$ For example, the existence of the limit in the case $m\left(x_{1}, x_{2}\right)=\left(x_{1}+x_{2}\right) / 2$ (see [5]) and consequently in the case of quasi-arithmetic means defined by $m\left(x_{1}, x_{2}\right)$ $=f^{-1}\left\{\left[f\left(x_{1}\right)+f\left(x_{2}\right)\right] / 2\right\},[1$, I. 4$]$. 